\title{
(6) OPEN ACCESS \\ Novel aneurysm neck reconstruction device: initial experience in an experimental preclinical bifurcation aneurysm model
}

ORIGINAL RESEARCH

\author{
Aquilla Turk, ${ }^{1}$ Raymond D Turner, ${ }^{2}$ Satoshi Tateshima, ${ }^{3}$ David Fiorella, ${ }^{4}$ \\ Kyung-Sool Jang, ${ }^{5}$ Imran Chaudry, ${ }^{1}$ Michael Kelly ${ }^{6}$
}

'Department of Radiology, Stroke and Cerebrovascular Center, Medical University of South Carolina, Charleston, South Carolina, USA

${ }^{2}$ Department of Neurosciences, Stroke and Cerebrovascular Center, Medical University of South Carolina, Charleston, South Carolina, USA

${ }^{3}$ Division of Interventional Neuroradiology, Department of Radiological Sciences, Ronald Reagan UCLA Medical Center, Los Angeles, California, USA

${ }^{4}$ Department of Neurological Surgery, Stony Brook University Medical Center, Stony Brook, New York, USA

${ }^{5}$ Department of Neurosurgery, Stroke and Cerebrovascular Center, The Catholic University of Korea, Incheon, Korea ${ }^{6}$ Division of Neurosurgery, Royal University Hospital, University of Saskatchewan, Saskatoon, Saskatchewan

\section{Correspondence to} Dr Aquilla Turk, Stroke and Cerebrovascular Center, Department of Radiology, Medical University of South Carolina, 96 Jonathan Lucas Street, Suite 337F CSB, Charleston, SC 29466, USA; turk@musc.edu

Received 14 February 2012 Revised 26 April 2012 Accepted 7 May 2012

Published Online First

3 June 2012

\begin{abstract}
Introduction Treatment of wide-necked bifurcation aneurysms often poses procedural and long-term outcome challenges. The initial preclinical experience with the Pulsar Vascular Aneurysm Neck Reconstruction Device (PVANRD) in a canine bifurcation model is described.
\end{abstract}

Methods Experimental bifurcation vein pouch aneurysms were surgically created in the carotid arteries of eight dogs. Endovascular coiling of the aneurysms with assistance of the PVANRD was performed in all cases with acute performance compared with Y-stenting.

Results Twelve devices were deployed in the eight cases. Deployment of the devices was straightforward and successfully protected the parent artery and maintained patency of the bifurcation in all cases, despite the use of oversized coils.

Conclusion The PVANRD is a novel bifurcation stent that facilitates treatment of wide-necked bifurcation aneurysms compared with currently available adjunctive devices.

\section{INTRODUCTION}

Endovascular treatment of intracranial aneurysms is rapidly becoming the preferred method of aneurysm treatment over open neurosurgical clipping. ${ }^{12}$ This is in large part due to advances in coil and stent technology that have improved safety and outcomes of the procedure. However, complex and broad-necked aneurysms continue to pose a challenge with a higher incidence of recurrence. ${ }^{3} 4$ These aneurysms also require advanced techniques such as balloon remodeling, dual microcatheter or stent-assisted coiling. ${ }^{5-10}$

Aneurysms located in a terminal or bifurcation anatomy create an even further difficulty as the anatomic and hematologic mechanical forces further increase the chance of coil compaction and aneurysm recurrence. When these aneurysms have broad necks, they often incorporate the adjacent branch vessels into the aneurysm neck which can create difficulty in treating the aneurysm without either occluding one of the outflow branches or leaving residual aneurysm. ${ }^{11} 12$ Early results suggest that more durable results may be obtained by using stents in conjunction with coils; however, current stents are engineered for sidewall aneurysm morphologies. ${ }^{13} 14$ The use of stents in bifurcation or terminal morphologies requires creative techniques such as Y-stenting where one stent passes through the interstices of the other stent, or sideby-side ('kissing') stents. ${ }^{14} 15$ These techniques are associated with increased difficulty of the procedure and peri-procedural risk. ${ }^{15}$ We present our early experience with a novel device designed for bifurcation aneurysm morphology in an experimental canine aneurysm model.

\section{METHODS}

The study was performed under an institutional animal committee approved protocol. Using a previously described surgical technique, bifurcation aneurysms were surgically created in eight dogs. The dogs were male beagles or hounds weighing between 9 and $29 \mathrm{~kg}$. All animals were treated with $81 \mathrm{mg}$ aspirin and $75 \mathrm{mg}$ clopidogrel daily beginning a week before the procedure. At least 3 weeks before evaluation of the device, vein pouch bifurcation aneurysms were surgically created using a well-described technique. ${ }^{16}$ All procedures were performed using standard endovascular techniques through femoral artery access. The dogs were heparinized with 1250 units heparin before commencing the procedure.

\section{Device}

The Pulsar Vascular Aneurysm Neck Reconstruction Device (PVANRD) is a novel approximately 0.002 -inch thick laser cut nitinol self-expanding device specifically shaped to fit within bifurcated arteries. It is designed to be deployed through a 0.027 -inch microcatheter at the bifurcation and abut the aneurysm ostium while remaining outside the aneurysm. The reconstructive scaffold area of the device or 'saddle' is oriented by opposing struts that align with the outflow branches to ensure preservation of those branches (figure 1). This results in a saddle-shaped web across the aneurysm neck that supports coils in the aneurysm while maintaining patency of the outflow branches. The proximal end of the device tapers to two struts that are anchored in the proximal parent vessel. Four radiopaque markers are present at the tips and midportion of the saddle to ensure appropriate orientation of the device at the aneurysm neck. There are four additional radiopaque markers oriented orthogonal to the saddle markers along the anchor legs to ensure appropriate expansion and alignment 


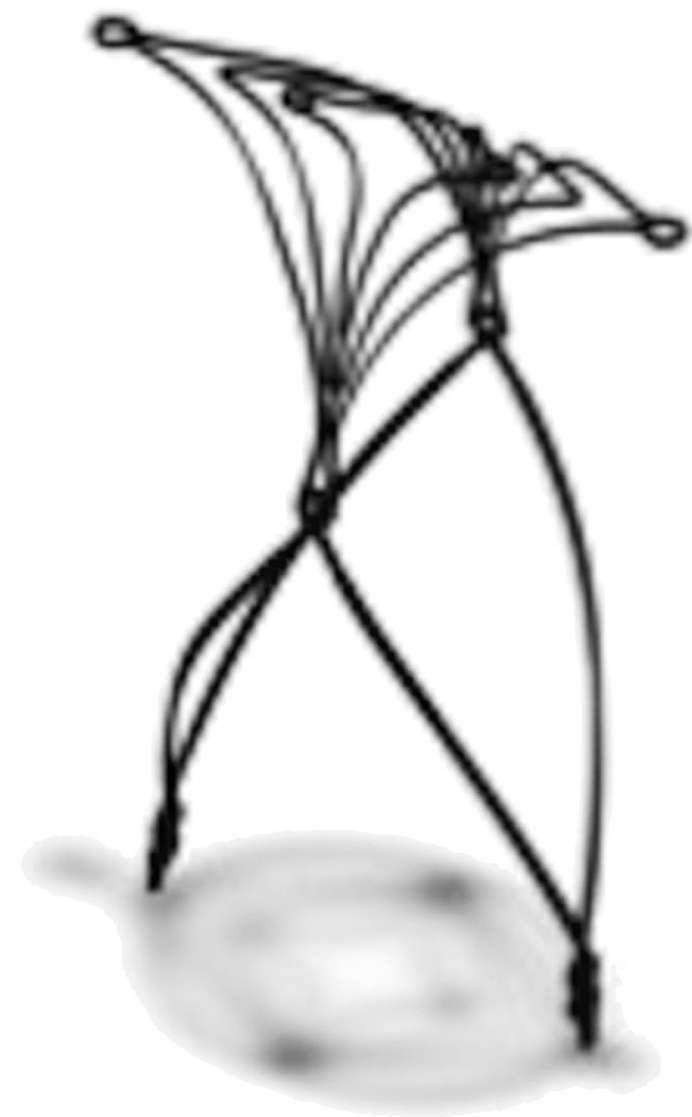

Figure 1 Pulsar Vascular Aneurysm Neck Reconstruction Device.

of the device. At the proximal end of the anchor legs is the electrolytic detachment zone, which is visible within the microcatheter prior to detachment.

\section{PROCEDURE}

Standard digital subtraction angiography was performed to characterize the aneurysm and obtain a working projection that best delineated the aneurysm neck and outflow branches and relation to the parent artery. The dimensions of the parent vessel, outflow branches and aneurysm were recorded. Through a 6 F sheath, a 0.027 -inch microcatheter was advanced to the aneurysm neck and the PVANRD deployed by unsheathing the device at the bifurcation. The device was placed so that the wings of the saddle were outside the aneurysm, aligned along the aneurysm neck and engaged with the proximal outflow branches. To position the arms of the saddle properly and to evaluate performance, the device was recaptured or repositioned several times. A technique deploying the device inside the aneurysm and then pulling back to engage the aneurysm neck was also used on some occasions. Each device deployment was performed by at least one of the authors. All authors deployed at least two devices in this study. All operators have significant clinical experience with Y-stenting to allow for educated comparison.

The technical performance of the PVANRD was evaluated as follows:

1. Overall performance: graded as acceptable or unacceptable.

2. Microwire traversal: a 0.010 -inch or 0.014 -inch guidewire was then advanced across the stent multiple times to assess for microwire interaction with the device. This was graded on a 3-point scale as same, better, or worse than that expected with Y-stenting in the operator's judgment.

3. Microcatheter traversal: a microcatheter of 0.017-inch internal diameter was manipulated over a 0.014-inch microwire through the interstices of the device and into the aneurysm. This was graded on a 3-point scale as same, better or worse than that expected with Y-stenting in the operator's judgment.

4. Coil retention: coil(s) were placed into the aneurysm to determine the ability of the device to maintain the coils within the aneurysm as well as to evaluate the stability of the device when oversized coils were placed (table 1).

Packing density was not measured. The goal of this project was not to achieve maximum packing density but rather to determine the performance of the device in stabilizing the framing and filling coils within the aneurysms. At the conclusion of the procedure the animals were killed and the devices explanted. Gross histology was not performed due to the acute nature of the study.

\section{RESULTS}

Twelve devices were placed in eight animals, each with a single experimental vein pouch bifurcation aneurysm. The devices were successfully delivered and deployed in all cases. Specific device performance, vessel and aneurysm dimensions and coils placed are listed in table 1.

In the first two animals the device was found to be undersized as the vessels were larger than expected. These two devices were noted to be unstable in the parent vessel. While the devices could have been removed at that time, the devices were detached to better understand their behavior in suboptimal conditions. The wings of the saddle prevented distal migration into the aneurysm. The first device was eventually pushed into the aneurysm, but this required some effort with a curved wire against the saddle. This device was then retrieved. A second device was more stable but, as expected due to its undersizing, poor apposition to the wall was found. Nonetheless, a $15 \mathrm{~cm} \times 30 \mathrm{~mm}$ coil was supported in the $13 \mathrm{~mm} \times 12 \mathrm{~mm}$ aneurysm by the device. The second animal similarly was found to have vessels larger than indicated for the test device. However, placement of two devices was performed. In both instances the device was noted to be undersized as expected and therefore 'floating' in the artery. However, due to the design of the device, the wings of the saddle prevented distal migration into the aneurysm. Significant wire manipulation was required to displace the PVANRD into the aneurysm. Although poorly positioned, it did adequately support a $15 \mathrm{~mm} \times 30 \mathrm{~cm}$ and a $24 \mathrm{~mm} \times 40 \mathrm{~cm}$ coil placed into a $10 \mathrm{~mm} \times 11 \mathrm{~mm}$ aneurysm despite the undersized device.

Design enhancements were subsequently made to the device to allow for use in a broader vessel size range for the subsequent studies. In the remaining six animals in the series the device was found to perform well with easy and accurate deployment and resheathing. It was easily manipulated so that appropriate positioning of the saddle across the aneurysm neck was possible in all cases. In most cases the device was resheathed and the orientation was rotated and changed several times to ensure design robustness. In all cases the device was stable and unable to be displaced with a guidewire or by navigating a microcatheter over a guidewire through it. The device was able to support placement of multiple significantly oversized coils into the aneurysm without prolapse of coil loops (figure 2). It was universally felt to be significantly easier to traverse than a Y-stent. The last device was found to be situated slightly caudal and in the bifurcation after its detachment. Subsequently, 


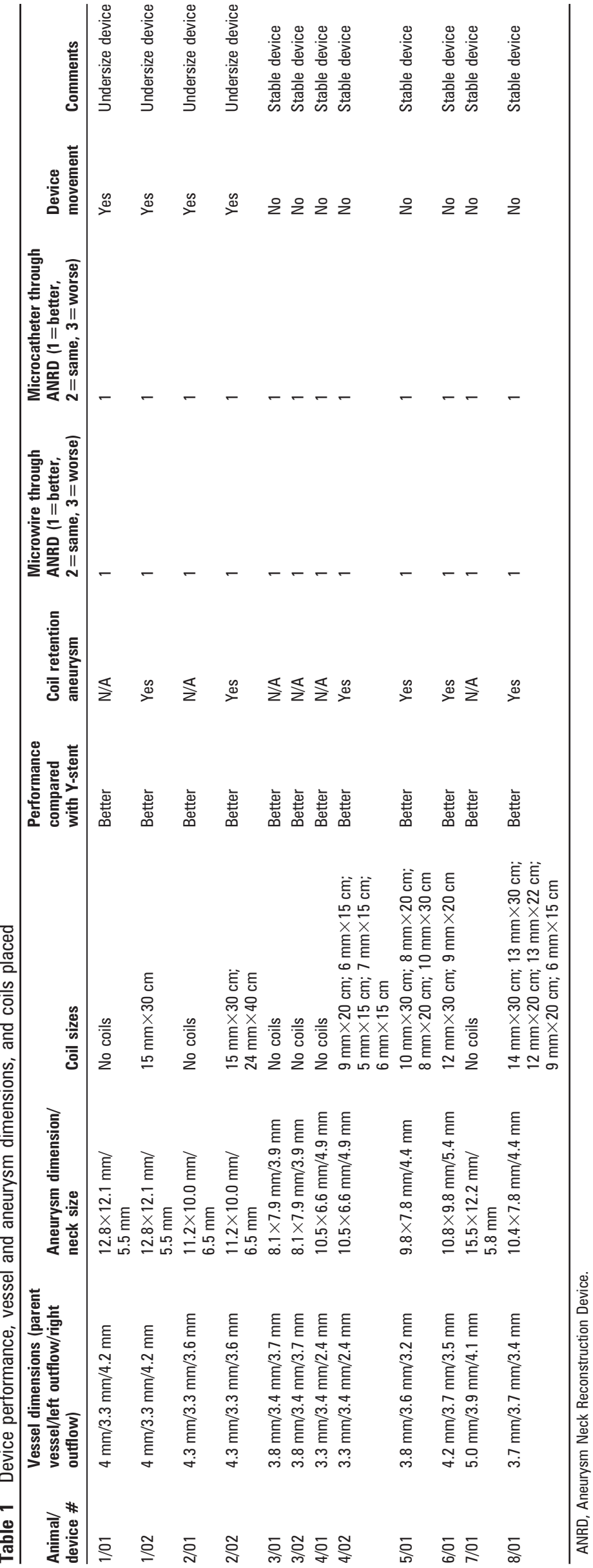



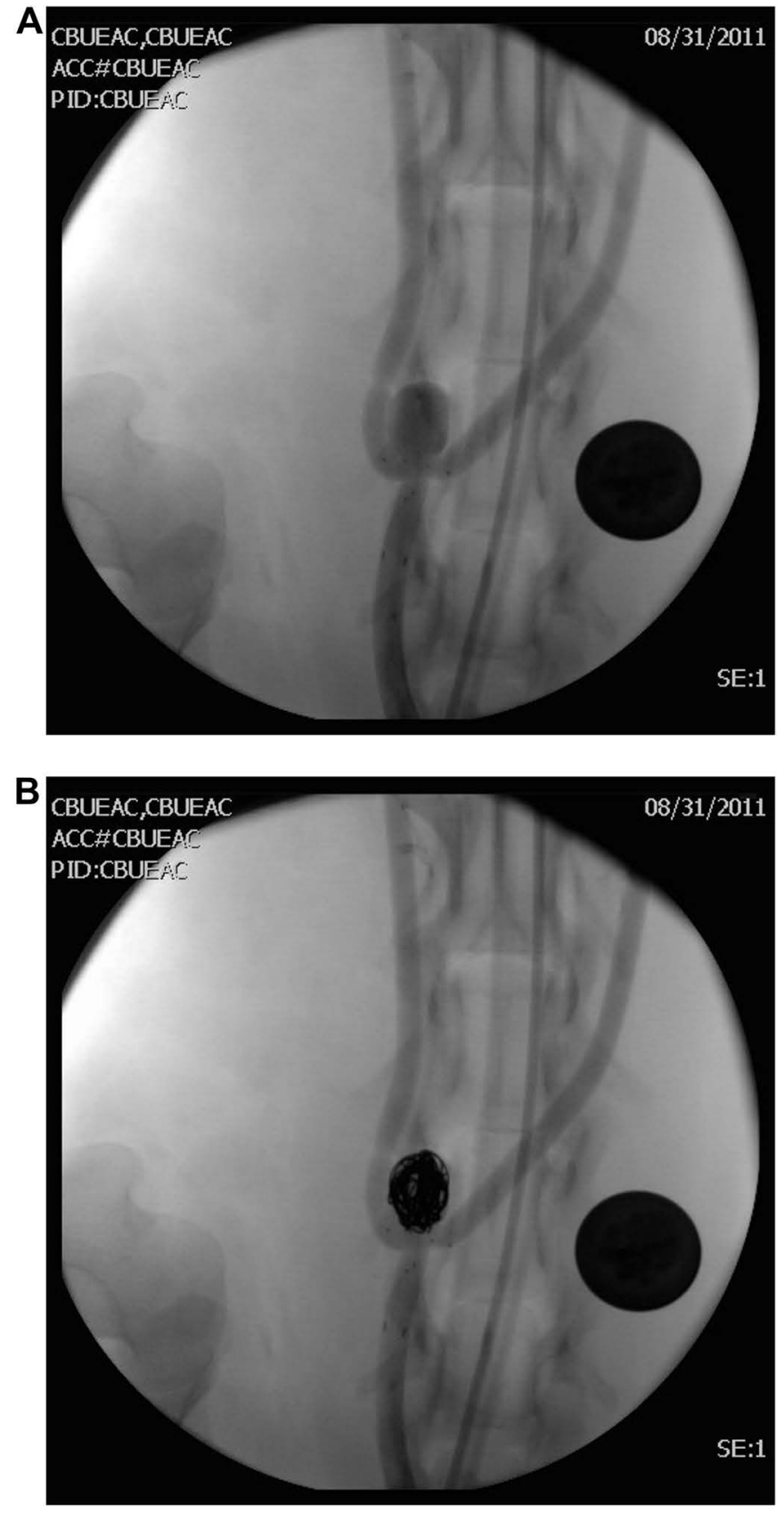

Figure 2 (A) Native digital subtraction angiography (DSA) image showing device deployed across aneurysm neck and microcatheter in aneurysm. (B) Native DSA image showing placement of a $14 \mathrm{~mm} \times 30$ $\mathrm{cm}$ and two $13 \mathrm{~mm} \times 30 \mathrm{~cm}$ coils with stable device position at aneurysm neck and no coil loop prolapse.

it could be advanced into an optimal position across the aneurysm ostium by careful engagement and manipulation with a curved guidewire, suggesting some degree of support by the wings of the saddle in the outflow branches.

\section{DISCUSSION}

The treatment of broad-necked aneurysms, especially those with a terminal or bifurcation morphology, continues to pose challenges for endovascular embolization. These lesions often require advanced techniques that can increase the risk of treatment and are also prone to increased risk of aneurysm recurrence. ${ }^{3-8}$ This is probably related to adapting tubular endovascular devices created for standard sidewall anatomy and using them in a creative fashion to treat bifurcation aneurysms. In this study we describe our experience with a novel device designed for bifurcation aneurysms which was found to offer superior performance, excellent stability and ease of use with robust coil support in experimental canine bifurcation aneurysms.

The primary challenge faced in treating broad-necked bifurcation aneurysms is the ability to achieve sufficient aneurysm occlusion with coils without compromising the outflow vessels. Dual or multiple microcatheter techniques are commonly used in many parts of the world, most prevalently in countries that do not have ready access to balloons. ${ }^{8-10}$ The foundation of the technique relies on complexing multiple coils together to form a stable basket by independently varying the orientation and placement of the complex shaped coils through multiple microcatheters. This allows the operator to optimize the features of complex coils to form a stable and ultra-complex basket that can then be filled in from the microcatheters at various points within the aneurysm. This technique is quite effective, but requires significant skill and does not provide definitive structural protection of the aneurysm neck or individual outflow branches.

Balloon remodeling allows the operator to expand the balloon and have it asymmetrically expand in a bifurcation to protect the aneurysm neck.' 1112 For more complex aneurysms, 'kissing balloons' can be used with a balloon in each outflow vessel, but this requires significant skill to manage two balloons simultaneously and coil the aneurysm. ${ }^{6}$ There are also challenges with access, as two guide catheters are often required for this procedure. This technique can be especially challenging in the posterior circulation where broad-necked basilar artery aneurysms may be the most prevalent. While balloons provide definitive vessel protection while they are inflated, there is a chance of coil loop prolapse when the balloon is deflated which could compromise the bifurcation or outflow branches.

Most recently, Y-stenting of these difficult lesions has shown the most promise for durable results but is associated with not insignificant procedural complications and technical challenges as well as increased cost. ${ }^{15}$ Y-stenting results in multiple layers of stents in the parent artery which may delay the healing process and increase the risk of thrombosis. This technique requires catheterization of one of the outflow branches followed by placement of a stent from the outflow branch distal to the aneurysm neck proximally into the parent vessel, then traversing through the interstices of the first stent and into the other outflow branch and deploying a second stent from that branch back into the first stent and parent artery. ${ }^{13}$ This can be technically very challenging, especially in large aneurysms with acute angulation of the outflow branch vessels in relation to the aneurysm ostium. Complications associated with this technique include prolapsing of the stent into the aneurysm while trying to advance a second stent for the final limb of the ' $Y$ '. This has resulted in a technique where some operators simply deploy a stent from proximally within the aneurysm down into the parent artery - that is, the 'waffle cone' technique. ${ }^{14}$ This does provide some structure for coils to engage and complex at the aneurysm neck, but does not protect the outflow branches.

There are challenges imposed by the study design, primarily related to the small number of aneurysms. However, this was a preliminary preclinical study for safety and device performance in a biologic model. The lack of long-term follow-up is also a limitation; however, this is related to the extreme mechanical movement of the animal neck with a fixed aneurysm implant which could artificially influence the histologic and angiographic outcome. The grading of device performance is subjective, but 
this was mitigated as much as possible by using multiple operators, all with significant clinical experience in treating these types of aneurysms.

The development of a true bifurcation aneurysm device may represent an important step forward in advancing the treatment of broad-necked bifurcation aneurysms. For a brief period the Tri-Span neck bridge device was available for human use outside the USA. ${ }^{17}$ This device similarly provided support at the aneurysm neck for coil retention in wide-necked aneurysms; however, the device was designed to be deployed inside the aneurysm and to be cohesive with the coil mass. This feature limited the use of the device only to those aneurysms that were taller than wide. The location of the device within the aneurysm is attractive in that antiplatelet drugs would not be required for its use; however, the structural support preventing compaction and recurrence may not be as robust as extra-aneurysmal device location. The current PVANRD device provides extra-aneurysmal structural support at the aneurysm neck with minimal intravascular surface exposure. The surface area of the PVANRD is approximately $85 \%$ less than the currently available tubular stents. This reduced intravascular exposure could potentially reduce the duration of clopidogrel and aspirin required for prophylaxis against thrombosis.

\section{CONCLUSION}

The present study demonstrates the feasibility of using the PVANRD to support coil embolization of bifurcation aneurysms. The device has some unique characteristics which may provide some advantages over conventional stents for the treatment of experimental bifurcation aneurysms.

Contributors Each author listed should receive authorship credit based on their material contribution to this article, their revision of this article, and their final approval of this article for submission to this journal.

Funding This work was supported by Pulsar Vascular Inc (grant number 88183)

Competing interests None.

Provenance and peer review Not commissioned; externally peer reviewed.

Open Access This is an Open Access article distributed in accordance with the Creative Commons Attribution Non Commercial (CC BY-NC 3.0) license, which permits others to distribute, remix, adapt, build upon this work non-commercially, and license their derivative works on different terms, provided the original work is properly cited and the use is non-commercial. See: http://creativecommons.org/ licenses/by-nc/3.0/

\section{REFERENCES}

1. Molyneux A, Kerr R, Stratton I, et al. International Subarachnoid Aneurysm Trial (ISAT) of neurosurgical clipping versus endovascular coiling in 2143 patients with ruptured intracranial aneurysms: a randomised trial. Lancet 2002;360:1267-74

2. Molyneux AJ, Kerr RS, Yu LM, et al. International Subarachnoid Aneurysm Trial (ISAT) Collaborative Group. International subarachnoid aneurysm trial (ISAT) of neurosurgical clipping versus endovascular coiling in 2143 patients with ruptured intracranial aneurysms: a randomised comparison of effects on survival, dependency, seizures, rebleeding, subgroups, and aneurysm occlusion. Lancet 2005;366:809-17.

3. Murayama Y, Nien YL, Duckwiler G, et al. Guglielmi detachable coil embolization of cerebral aneurysms: 11 years' experience. J Neurosurg 2003;98:959-66.

4. Zubillaga F A, Guglielmi G, Viñuela F, et al. Endovascular occlusion of intracranial aneurysms with electrically detachable coils: correlation of aneurysm neck size and treatment results. AJNR Am J Neuroradiol 1994;15:815-20.

5. Bodily KD, Cloft HJ, Lanzino G, et al. Stent-assisted coiling in acutely ruptured intracranial aneurysms: a qualitative, systematic review of the literature. AJNR Am J Neuroradiol 2011;32:1232-6.

6. Kelly ME, Gonugunta V, Woo HH, et al. Double-balloon trapping technique for embolization of a large wide-necked superior cerebellar artery aneurysm: case report. Neurosurgery 2008:63(4 Suppl 2):291-2.

7. Pierot L, Cognard C, Anxionnat R, et al. CLARITY Investigators. Remodeling technique for endovascular treatment of ruptured intracranial aneurysms had a higher rate of adequate postoperative occlusion than did conventional coil embolization with comparable safety. Radiology 2011;258:546-53

8. Baxter B, Rosso D, Lownie S. Double microcatheter technique for detachable coil treatment of large, wide-necked intracranial aneurysms. AJNR Am J Neuroradiol 1998;19:1176-8.

9. Setton A, Prestigiacomo C, Niimi Y, et al. Dual microcatheter technique for the treatment of aneurysms with relatively wide necks. Neurosurgery 2006;58:407

10. Horowitz M, Gupta R, Jovin T. The dual catheter technique for coiling of wide-necked cerebral aneurysms. An under-reported method. Interv Neuroradiol 2005;11:155-60.

11. Lazzaro MA, Darkhabani Z, Zaidat 00, et al. Initial experience with the coaxial dual-lumen ascent balloon catheter for wide-neck aneurysm coil embolization. Front Neurol 2011:2:52

12. Pukenas B, Albuquerque FC, Weigele JB, et al. Use of a new double-lumen balloon catheter for single-catheter balloon-assisted coil embolization of intracranial aneurysms: technical note. Neurosurgery 2011;69(1 Suppl Operative):ons8-12; discussion ons12-13.

13. Thorell WE, Chow MM, Woo HH, et al. Y-configured dual intracranial stent-assisted coil embolization for the treatment of wide-necked basilar tip aneurysms. Neurosurgery 2005;56:1035-40.

14. Yang TH, Wong HF, Yang MS, et al. "Waffle cone" technique for intra/extraaneurysmal stent placement for the treatment of complex and wide-necked bifurcation aneurysm. Interv Neuroradiol 2008;14(Suppl 2):49-52.

15. Spiotta AM, Gupta R, Fiorella D, et al. Mid-term results of endovascular coiling of wide-necked aneurysms using double stents in a $\mathrm{Y}$ configuration. Neurosurgery 2011;69:421-9.

16. German WJ, Black PW. Experimental production of carotid aneurysms. N Engl J Med 1954;250:104-6.

17. Raymond J, Guilbert F, Roy D. Neck-bridge device for endovascular treatment of wide-neck bifurcation aneurysms: initial experience. Radiology 2001;221:318-26. 